\title{
Facile Migratory Insertion of a N-Heterocyclic Carbene into a Ruthenium- Carbon Double Bond - A New Type of Reaction of a NHC Ligand
}

Eva Becker, Verena Stingl, Georg Dazinger, Michael Puchberger, Kurt Mereiter, and Karl Kirchner*

\section{Supporting Information}

\section{Experimental Section}

General Techniques. All manipulations were performed under an inert atmosphere of argon by using Schlenk techniques. All chemicals were standard reagent grade and used without further purification. The solvents were purified according to standard procedures. ${ }^{1}$ The deuterated solvents were purchased from Aldrich and dried over $4 \AA$ molecular sieves. $\left[\mathrm{RuCp}\left(\mathrm{CH}_{3} \mathrm{CN}\right)_{3}\right] \mathrm{PF}{ }_{6}$ and IPr.'HCl were prepared according to the literature. ${ }^{2,3}{ }^{1} \mathrm{H}$ and ${ }^{13} \mathrm{C}\left\{{ }^{1} \mathrm{H}\right\}$ NMR spectra were recorded on a Bruker AVANCE-250 spectrometer operating at 250.13 and $62.86 \mathrm{MHz}$, respectively, and were referenced to $\mathrm{SiMe}_{4}$. ${ }^{1} \mathrm{H}$ and ${ }^{13} \mathrm{C}\left\{{ }^{1} \mathrm{H}\right\}$ NMR signal assignments were confirmed by ${ }^{1} \mathrm{H}$-COSY, 135-DEPT, and HSQC $\left({ }^{1} \mathrm{H}^{13} \mathrm{C}\right)$ experiments.

[RuCp(IPr') $\left.\left(\mathrm{CH}_{3} \mathrm{CN}\right)_{2}\right] \mathrm{PF}_{6}$ (1). A suspension of IPr'. $\mathrm{HCl}(690 \mathrm{mg}, 1.62 \mathrm{mmol})$ in $20 \mathrm{~mL}$ of toluene was cooled to $-40^{\circ} \mathrm{C}$ and $n$-BuLi $(0.65 \mathrm{~mL}$ of a $2.6 \mathrm{M}$ solution in $n$-hexane) was added via syringe. The mixture was stirred for $30 \mathrm{~min}$ at room temperature. $\mathrm{LiCl}$ was removed by filtration. This solution was then slowly added to a stirred solution of $\left[\mathrm{RuCp}\left(\mathrm{CH}_{3} \mathrm{CN}\right)_{3}\right] \mathrm{PF}_{6}(350 \mathrm{mg}, 0.81 \mathrm{mmol})$ in THF $(10 \mathrm{~mL})$. The solution was stirred at room temperature for $30 \mathrm{~min}$. After removal of the solvent under vacuum the remaining brown solid was dissolved in $2 \mathrm{~mL}$ of acetonitrile and purified by column chromatography (neutral $\mathrm{Al}_{2} \mathrm{O}_{3}$, eluent: $\mathrm{CH}_{3} \mathrm{CN}$ ). 2 was isolated as a yellow microcrystalline solid. Yield: $78 \%$ (486 mg). Anal. Calcd. for $\mathrm{C}_{36} \mathrm{H}_{47} \mathrm{~F}_{6} \mathrm{~N}_{4} \mathrm{PRu}$ : C, 55.24; $\mathrm{H}, 6.05 ; \mathrm{N}, 7.16$. Found: C, 55.44; $\mathrm{H}$, 6.11; N, 7.25. ${ }^{1} \mathrm{H}$ NMR $\left(\delta\right.$, acetone- $\left.\mathrm{d}_{6}, 20^{\circ} \mathrm{C}\right): 7.56\left(\mathrm{~s}, 2 \mathrm{H}, \mathrm{IPr}^{\mathrm{i}} \mathrm{CH}\right), 7.55\left(\mathrm{t}, \mathrm{J}_{H H}=8.1 \mathrm{~Hz}, 2 \mathrm{H}, \mathrm{Ar}^{4}\right), 7.43$ $\left(\mathrm{d}, J_{H H}=8.1,4 \mathrm{H}, \mathrm{Ar}^{3,5}\right), 3.85(\mathrm{~s}, 5 \mathrm{H}, \mathrm{Cp}), 2,92\left(\mathrm{~m}, J_{H H}=6.9 \mathrm{~Hz}, 4 \mathrm{H}, \mathrm{Pr}^{\mathrm{i}}-\mathrm{CH}\right), 2.23\left(\mathrm{~s}, 6 \mathrm{H}, \mathrm{CH}_{3} \mathrm{CN}\right), 1.35$ (d, $\left.J_{H H}=6.9 \mathrm{~Hz}, 12 \mathrm{H}, \operatorname{Pr}^{\mathrm{i}}-\mathrm{CH}_{3}\right), 1.18\left(\mathrm{~d}, \mathrm{~J}_{H H}=6.9 \mathrm{~Hz}, 12 \mathrm{H}, \operatorname{Pr}^{\mathrm{i}}-\mathrm{CH}_{3}\right) \cdot{ }^{13} \mathrm{C}\left\{{ }^{1} \mathrm{H}\right\}$ NMR $\left(\delta\right.$, acetone- $\mathrm{d}_{6}$, $\left.20^{\circ} \mathrm{C}\right): 185.2\left(\operatorname{Irr}^{\mathrm{i}-1}\right), 146.3\left(\mathrm{Ar}^{2,6}\right), 137.9\left(\operatorname{Ar}^{1}\right), 130.4\left(\mathrm{Ar}^{4}\right), 125.9\left(\operatorname{Pr}^{\mathrm{i}-3,4}\right), 123.8\left(\mathrm{Ar}^{3,5}\right), 73.0(\mathrm{Cp}), 28.4$ (Pri- $\mathrm{CH}), 24.9\left(\mathrm{Pr}^{\mathrm{i}}-\mathrm{CH}_{3}\right), 21.8\left(\mathrm{Pr}^{\mathrm{i}}-\mathrm{CH}_{3}\right)$.

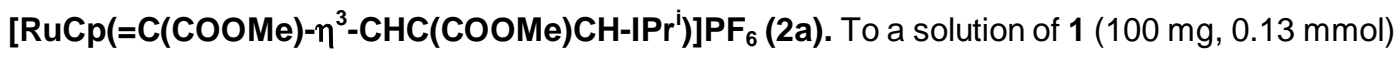
in $5 \mathrm{~mL} \mathrm{CH} \mathrm{Cl}_{2}$ methyl propiolate $(35 \mu \mathrm{L}, 0.38 \mathrm{mmol})$ was added. The purple solution was stirred at 
room temperature for $30 \mathrm{~min}$. After the solvent was removed the remaining solid was purified by column chromatography (neutral $\mathrm{Al}_{2} \mathrm{O}_{3}$, eluent: $\mathrm{Et}_{2} \mathrm{O} / \mathrm{CH}_{2} \mathrm{Cl}_{2}$ 1:1). Yield: $93 \%$ (103 mg). Anal. Calcd. For $\mathrm{C}_{40} \mathrm{H}_{49} \mathrm{~F}_{6} \mathrm{~N}_{2} \mathrm{O}_{4} \mathrm{PRu}$ : C, 55.36; $\mathrm{H}, 5.69 ; \mathrm{N}, 3.23$. Found: $\mathrm{C}, 55.47 ; \mathrm{H}, 5.76 ; \mathrm{N}, 3.19 .{ }^{1} \mathrm{H}$ NMR $(\delta$, acetone- $\left.\mathrm{d}_{6}, 20^{\circ} \mathrm{C}\right): 7.94\left(\mathrm{~s}, 2 \mathrm{H}, \mathrm{IPr}^{\mathrm{i}} \mathrm{CH}\right), 7.53-7.78(\mathrm{~m}, 6 \mathrm{H}, \mathrm{Ar}), 6.08\left(\mathrm{~s}, 1 \mathrm{H}, \mathrm{H}^{4}\right), 5.75\left(\mathrm{~s}, 1 \mathrm{H}, \mathrm{H}^{2}\right), 4.78$ (s, 5H, Cp), $3.88\left(\mathrm{~s}, 3 \mathrm{H}, \mathrm{COOCH}_{3}\right), 3.53\left(\mathrm{~s}, 3 \mathrm{H}, \mathrm{COOCH}_{3}\right), 2.59\left(\mathrm{~m}, 4 \mathrm{H}, \operatorname{Pr}^{\mathrm{i}}-\mathrm{CH}\right), 1.35-1.10(\mathrm{~m}, 24 \mathrm{H}$, $\left.\operatorname{Pr}^{\mathrm{i}}-\mathrm{CH}_{3}\right) .{ }^{13} \mathrm{C}\left\{{ }^{1} \mathrm{H}\right\}$ NMR $\left(\delta\right.$, acetone- $\left.\mathrm{d}_{6}, 20^{\circ} \mathrm{C}\right): 237.4\left(\mathrm{Ru}=\mathrm{C}^{1}\right), 172.6\left(\mathrm{C}^{1} \mathrm{COOMe}\right), 168.6\left(\mathrm{C}^{3} \mathrm{COOMe}\right)$, 153.6 $\left(\operatorname{IPr}^{\mathrm{i}-1}\right), 145.2\left(\operatorname{Ar}^{1}\right), 145.1\left(\operatorname{Ar}^{2,6}\right), 126.8\left(\operatorname{IPr}^{\mathrm{i}-3,4}\right), 125.6\left(\operatorname{Ar}^{3,5}\right), 125.3\left(\operatorname{Ar}^{4}\right), 88.2\left(C^{2}\right), 86.8(\mathrm{Cp})$, $86.4\left(\mathrm{C}^{3}\right), 52.2\left(\mathrm{C}^{1} \mathrm{COOCH}_{3}\right), 51.8\left(\mathrm{C}^{3} \mathrm{COOCH}_{3}\right), 30.3\left(\mathrm{C}^{4}\right), 29.3\left(\mathrm{Pr}^{\mathrm{i}}-\mathrm{CH}\right), 29.0\left(\operatorname{Pr}^{\mathrm{i}}-\mathrm{CH}\right), 25.3\left(\operatorname{Pr}^{\mathrm{i}}-\right.$ $\left.\mathrm{CH}_{3}\right), 23.7\left(\mathrm{Pr}^{\mathrm{i}}-\mathrm{CH}_{3}\right), 22.9\left(\mathrm{Pr}^{\mathrm{i}}-\mathrm{CH}_{3}\right), 21.4\left(\operatorname{Pr}^{\mathrm{i}}-\mathrm{CH}_{3}\right)$.

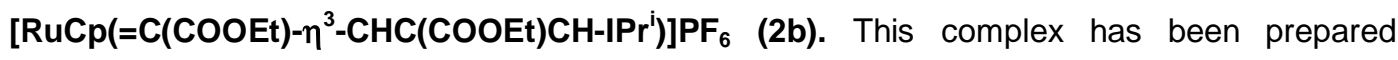
analogously to 2 a with 1 (100 $\mathrm{mg}, 0.13 \mathrm{mmol}$ ) and ethyl propiolate (39 $\mu \mathrm{L}, 0.38 \mathrm{mmol}$ ) as starting materials. Yield: 94 \% (108 mg). Anal. Calcd. For $\mathrm{C}_{42} \mathrm{H}_{53} \mathrm{~F}_{6} \mathrm{~N}_{2} \mathrm{O}_{4} \mathrm{PRu}: \mathrm{C}, 56.31 ; \mathrm{H}, 5.96 ; \mathrm{N}, 3.13$. Found: C, 56.44; H, 6.04; N, 3.21. ${ }^{1} \mathrm{H}$ NMR $\left(\delta\right.$, acetone- $\left.\mathrm{d}_{6}, 20^{\circ} \mathrm{C}\right): 7.94(\mathrm{~s}, 2 \mathrm{H}, \mathrm{IPr} \mathrm{C} H), 7.51-7.69(\mathrm{~m}$, $6 \mathrm{H}, \mathrm{Ar}), 6.10\left(\mathrm{~s}, 1 \mathrm{H}, \mathrm{H}^{4}\right), 5.75\left(\mathrm{~s}, 1 \mathrm{H}, \mathrm{H}^{2}\right), 4.77(\mathrm{~s}, 5 \mathrm{H}, \mathrm{Cp}), 4.47-3.86\left(\mathrm{~m}, 4 \mathrm{H}, \mathrm{CH}_{2}\right), 2.59\left(\mathrm{~m}, 4 \mathrm{H}, \mathrm{Pr}^{\mathrm{i}}-\right.$ $\mathrm{CH}), 1.35-1.10\left(\mathrm{~m}, 24 \mathrm{H}, \operatorname{Pr}-\mathrm{CH}_{3}\right) \cdot{ }^{13} \mathrm{C}\left\{{ }^{1} \mathrm{H}\right\} \operatorname{NMR}\left(\delta\right.$, acetone- $\left.\mathrm{d}_{6}, 20^{\circ} \mathrm{C}\right): 238.1\left(\mathrm{Ru}=\mathrm{C}^{1}\right), 172.4$ (C ${ }^{3}$ COOEt), 168.1 (C COOEt), $153.7\left(\operatorname{IPr}^{1-1}\right), 146.1\left(\operatorname{Ar}^{1}\right), 145.2\left(\operatorname{Ar}^{2,6}\right), 132.2\left(\operatorname{Ar}^{3,5}\right), 125.3\left(\operatorname{Ar}^{4}\right), 124.8$ (IPri-3,4), 88.0 $\left(\mathrm{C}^{2}\right)$, $86.7(\mathrm{Cp}), 86.6\left(\mathrm{C}^{3}\right), 61.8\left(\mathrm{C}^{1} \mathrm{COOCH}_{2}\right), 61.4\left(\mathrm{C}^{3} \mathrm{COOCH}_{2}\right), 30.5\left(\mathrm{C}^{4}\right), 29.0\left(\mathrm{Pr}^{\mathrm{i}}-\right.$ $\mathrm{CH}), 25.2\left(\mathrm{Pr}^{\mathrm{i}}-\mathrm{CH}_{3}\right), 23.7\left(\mathrm{Pr}^{\mathrm{i}}-\mathrm{CH}_{3}\right), 22.9\left(\mathrm{Pr}^{\mathrm{i}}-\mathrm{CH}_{3}\right), 21.5\left(\mathrm{Pr}^{\mathrm{i}}-\mathrm{CH}_{3}\right), 13.7\left(\mathrm{C}^{1} \mathrm{COO} \mathrm{CH}_{2} \mathrm{CH}_{3}\right)$, 13.3 $\left(\mathrm{C}^{3} \mathrm{COO} \mathrm{CH}_{2} \mathrm{CH}_{3}\right)$.

$\left[\mathrm{RuCp}\left(=\mathrm{C}(\mathrm{COMe})-\eta^{3}-\mathrm{CHC}(\mathrm{COMe}) \mathrm{CH}-\mathrm{IPr}^{\mathrm{i}}\right)\right] \mathrm{PF}_{6} \quad(\mathbf{2 c})$. This complex has been prepared analogously to $2 \mathrm{a}$ with 1 (100 mg, $0.13 \mathrm{mmol})$ and 3-Butin-3-on $(30 \mu \mathrm{L}, 0.38 \mathrm{mmol}$ ) as starting materials. Yield: $89 \%$ (94 mg). Anal. Calcd. For $\mathrm{C}_{40} \mathrm{H}_{49} \mathrm{~F}_{6} \mathrm{~N}_{2} \mathrm{O}_{4} \mathrm{PRu}$ : C, 57.48; H, 5.91; N, 3.35. Found: $\mathrm{C}, 57.34 ; \mathrm{H}, 5.80 ; \mathrm{N}, 3.41 .{ }^{1} \mathrm{H} N M R\left(\delta, \mathrm{CD}_{2} \mathrm{Cl}_{2}, 20^{\circ} \mathrm{C}\right): 7.66\left(\mathrm{t}, \mathrm{J}_{H H}=7.8 \mathrm{~Hz}, 2 \mathrm{H}, \mathrm{Ar}^{4}\right), 7.44\left(\mathrm{~d}, J_{H H}=8.1\right.$, $\left.4 \mathrm{H}, \mathrm{Ar}^{3,5}\right), 7.13\left(\mathrm{~s}, 2 \mathrm{H}, \mathrm{IPr}^{\mathrm{i}} \mathrm{CH}\right), 5.98\left(\mathrm{~s}, 1 \mathrm{H}, \mathrm{H}^{4}\right), 5.38\left(\mathrm{~s}, 1 \mathrm{H}, \mathrm{H}^{2}\right), 4.63(\mathrm{~s}, 5 \mathrm{H}, \mathrm{Cp}), 2.41\left(\mathrm{~m}, \mathrm{~J}_{H H}=6.8\right.$, $\left.4 \mathrm{H}, \mathrm{Pr}^{\mathrm{i}}-\mathrm{CH}\right), 2.40\left(\mathrm{~s}, 3 \mathrm{H}, \mathrm{COCH}_{3}\right), 1.87\left(\mathrm{~s}, 3 \mathrm{H}, \mathrm{COCH}_{3}\right), 1.31$ (d, $\left.\mathrm{J}_{H H}=6.8 \mathrm{~Hz}, 12 \mathrm{H}, \operatorname{Pr}^{\mathrm{i}}-\mathrm{CH}_{3}\right), 1.23$ (d, $\left.J_{H H}=6.8 \mathrm{~Hz}, 12 \mathrm{H}, \operatorname{Pr}^{\mathrm{i}}-\mathrm{CH}_{3}\right) \cdot{ }^{13} \mathrm{C}\left\{{ }^{1} \mathrm{H}\right\} \operatorname{NMR}\left(\delta, \mathrm{CD}_{2} \mathrm{Cl}_{2}, 20^{\circ} \mathrm{C}\right): 251.2\left(\mathrm{Ru}=\mathrm{C}^{1}\right), 209.0\left(\mathrm{C}^{1} \mathrm{COMe}\right), 199.0$ $\left(\mathrm{C}^{3} \mathrm{COMe}\right), 154.1\left(\operatorname{IPr}^{\mathrm{i}-1}\right), 145.0\left(\mathrm{Ar}^{2,6}\right), 136.9\left(\operatorname{IPr}^{\mathrm{i}-3,4}\right), 132.4\left(\mathrm{Ar}^{3,5}\right), 129.4\left(\operatorname{Ar}^{1}\right), 124.9\left(\operatorname{Ar}^{4}\right), 91.7\left(\mathrm{C}^{2}\right)$, $86.7(\mathrm{Cp}), 84.7\left(\mathrm{C}^{3}\right), 29.1\left(\mathrm{COCH}_{3}\right), 28.5\left(\mathrm{Pr}^{\mathrm{i}}-\mathrm{CH}\right), 28.1\left(\mathrm{Pr}^{\mathrm{i}}-\mathrm{CH}\right), 25.7\left(\mathrm{C}^{4}\right), 24.0\left(\right.$ Pri $\left.^{\mathrm{i}}-\mathrm{CH}_{3}\right), 23.5\left(\operatorname{Pr}^{\mathrm{i}}-\right.$ $\left.\mathrm{CH}_{3}\right)$. 
$\left[\mathrm{RuCp}\left(=\mathrm{CH}-\eta^{3}-\mathrm{C}\left(\mathrm{CH}_{2}\right)_{4} \mathrm{CCH}-\mathrm{IPr}^{\mathrm{i}}\right)\right] \mathrm{PF}_{6}(\mathbf{2 d})$. This complex has been prepared analogously to $2 \mathrm{a}$ with 1 (100 mg, $0.13 \mathrm{mmol}$ ) and 1,7-octadiyne (17 $\mu \mathrm{L}, 0.13 \mathrm{mmol})$ as starting materials. Yield: $94 \%$ (96 mg). Anal. Calcd. For $\mathrm{C}_{40} \mathrm{H}_{51} \mathrm{~F}_{6} \mathrm{~N}_{2} \mathrm{PRu}$ : C, 59.62; H, 5.91; N, 3.35. Found: C, 59.54; $\mathrm{H}, 6.08$; $\mathrm{N}$, 3.48. ${ }^{1} \mathrm{H}$ NMR $\left(\delta, \mathrm{CD}_{2} \mathrm{Cl}_{2}, 20^{\circ} \mathrm{C}\right): 12.15\left(\mathrm{~s}, 1 \mathrm{H}, \mathrm{H}^{1}\right), 7.53\left(\mathrm{t}, \mathrm{J}_{H H}=7.8 \mathrm{~Hz}, 2 \mathrm{H}, \mathrm{Ar}^{4}\right), 7.44\left(\mathrm{~d}, \mathrm{~J}_{H H}=7.8\right.$, $\left.4 \mathrm{H}, \mathrm{Ar}^{3,5}\right), 7.02(\mathrm{~s}, 2 \mathrm{H}, \mathrm{IPr} \mathrm{CH}), 5.07\left(\mathrm{~s}, 1 \mathrm{H}, \mathrm{H}^{4}\right), 4.48(\mathrm{~s}, 5 \mathrm{H}, \mathrm{Cp}), 2.43\left(\mathrm{~m}, \mathrm{~J}_{H H}=6.8,4 \mathrm{H}, \operatorname{Pr}^{\mathrm{i}}-\mathrm{CH}\right), 2.28$ - $2.00\left(\mathrm{~m}, 4 \mathrm{H}, \mathrm{CH}_{2}\right), 1.89-1.65\left(\mathrm{~m}, 2 \mathrm{H}, \mathrm{CH}_{2}\right), 1.47-1.01\left(\mathrm{~m}, 2 \mathrm{H}, \mathrm{CH}_{2}\right), 1.62\left(\mathrm{~d}, \mathrm{~J}_{\mathrm{HH}}=6.8 \mathrm{~Hz}, 6 \mathrm{H}, \mathrm{Pr}^{\mathrm{i}}-\right.$ $\left.\mathrm{CH}_{3}\right), 1.53\left(\mathrm{~d}, \mathrm{~J}_{H H}=6.8 \mathrm{~Hz}, 6 \mathrm{H}, \mathrm{Pr}^{\mathrm{i}}-\mathrm{CH}_{3}\right), 1.15\left(\mathrm{~d}, \mathrm{~J}_{H H}=3.6 \mathrm{~Hz}, 6 \mathrm{H}, \operatorname{Pr}^{\mathrm{i}}-\mathrm{CH}_{3}\right), 1.12\left(\mathrm{~d}, \mathrm{~J}_{H H}=3.8 \mathrm{~Hz}, 6 \mathrm{H}\right.$, $\left.\operatorname{Pr}^{\mathrm{i}}-\mathrm{CH}_{3}\right) .{ }^{13} \mathrm{C}\left\{{ }^{1} \mathrm{H}\right\} \operatorname{NMR}\left(\delta, \mathrm{CD}_{2} \mathrm{Cl}_{2}, 20^{\circ} \mathrm{C}\right): 245.2\left(\mathrm{Ru}=\mathrm{C}^{1}\right), 155.4\left(\operatorname{Ir}^{\mathrm{i}-1}\right), 145.8\left(\mathrm{Ar}^{2,6}\right), 144.7\left(\mathrm{Ar}^{2,6}\right)$, $137.0\left(\operatorname{IPr}^{\mathrm{i}-3,4}\right), 132.4\left(\mathrm{Ar}^{3,5}\right), 132.1\left(\mathrm{Ar}^{3,5}\right), 131.2\left(\mathrm{Ar}^{1}\right), 129.5\left(\operatorname{Ar}^{1}\right), 125.3\left(\operatorname{Ar}^{4}\right), 125.0\left(\operatorname{Ar}^{4}\right), 102.5\left(\mathrm{C}^{2}\right)$, $100.7\left(\mathrm{C}^{3}\right)$, $84.2(\mathrm{Cp}), 34.7\left(\mathrm{CH}_{2}\right), 30.3\left(\mathrm{C}^{4}\right), 29.6\left(\right.$ Pri $\left.^{\mathrm{i}} \mathrm{CH}\right), 29.2\left(\mathrm{Pr}^{\mathrm{i}}-\mathrm{CH}\right), 26.0\left(\mathrm{Pr}^{\mathrm{i}}-\mathrm{CH}\right), 25.9\left(\mathrm{CH}_{2}\right)$, $25.4(\operatorname{Pr}-\mathrm{CH}), 22.0\left(\mathrm{CH}_{2}\right), 21.9\left(\operatorname{Pr}^{\mathrm{i}}-\mathrm{CH}_{3}\right), 21.8\left(\operatorname{Pr}^{\mathrm{i}}-\mathrm{CH}_{3}\right), 21.0\left(\mathrm{CH}_{2}\right)$.

Reaction of 1 with 1,7-octadiyne in $\mathrm{CD}_{2} \mathrm{Cl}_{2}$. A $5 \mathrm{~mm}$ NMR Young-tube was charged with 1 (30 $\mathrm{mg}, 0.040 \mathrm{mmol})$ in $\mathrm{CD}_{2} \mathrm{Cl}_{2}(0.5 \mathrm{~mL})$ and cooled to $-20^{\circ} \mathrm{C}$. Upon addition of 1,7 -octadiyne $(5.2 \mu \mathrm{L}, 0.04$ mmol) the reaction was monitored by ${ }^{1} \mathrm{H}$ and ${ }^{13} \mathrm{C}$ NMR spectroscopy. After 14 hours at this temperature two signals in a ratio of approximately $1: 1$ at $17.08 \mathrm{ppm}$ and $12.15 \mathrm{ppm}(\mathbf{2 d})$ were detected in the ${ }^{1} \mathrm{H}$ NMR spectrum. $A{ }^{1} \mathrm{H}^{13} \mathrm{C}-\mathrm{HSQC}$ spectrum showed the corresponding carbon signals at 298.0 and $244.9 \mathrm{ppm}$. After warming the solution to room temperature the signal at 17.08 dissapeared within one hour and quantitaive formation of $\mathbf{2} \mathbf{d}$ was observed. 


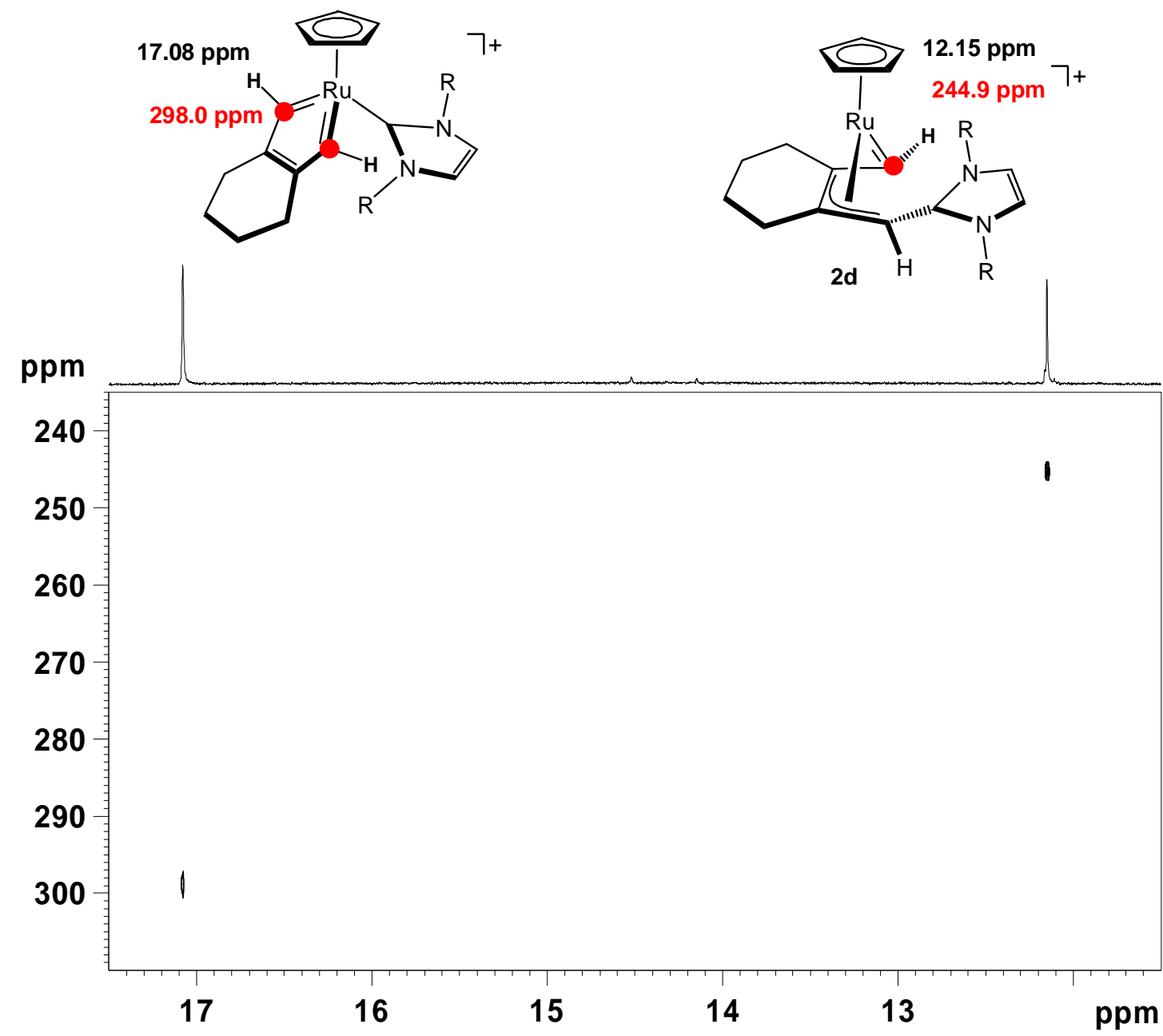

Figure S1. ${ }^{1} \mathrm{H}-{ }^{13} \mathrm{C}-\mathrm{HSQC}$ NMR spectrum of the reaction of 1 with 1,7 -octadiyne in $\mathrm{CD}_{2} \mathrm{Cl}_{2}$ at $-20^{\circ} \mathrm{C}$ after $14 \mathrm{~h}$ of reaction time showing the formation of a ruthenacyclopentatriene intermediate together with the final product $\mathbf{2 d}$.

X-ray Structure Determination. X-ray data for $\mathbf{2 b} \cdot \mathrm{CH}_{2} \mathrm{Cl}_{2}$ were collected on a Bruker Smart CCD area detector diffractometer using graphite-monochromated Mo Ka radiation $(\lambda=0.71073 \AA)$ and $0.3^{\circ} \omega$-scan frames covering a complete sphere of the reciprocal space $\left(\theta_{\max }=30^{\circ}\right)$. Corrections for absorption, $\lambda / 2$ effects, and crystal decay were applied. ${ }^{4}$ The structure was solved by direct methods using the program SHELXS97. ${ }^{5}$ Structure refinement on $F^{2}$ was carried out with the program SHELXL97. ${ }^{13}$ All non-hydrogen atoms were refined anisotropically. The hydrogen atoms $\mathrm{H}(7)$ and $\mathrm{H}(9)$ of the allyl system were refined without restraints. All other hydrogen atoms were inserted in idealized positions and were refined riding with the atoms to which they were bonded.

Crystal data of $\mathbf{2 b} \cdot \mathrm{CH}_{2} \mathrm{Cl}_{2}: \mathrm{C}_{43} \mathrm{H}_{55} \mathrm{Cl}_{2} \mathrm{~F}_{6} \mathrm{~N}_{2} \mathrm{O}_{4} \mathrm{PRu}, M_{r}=980.83$, orthorhombic, space group Pna2 1 (no. 33), $T=100(2) \mathrm{K}, a=19.1884(12) \AA, b=12.1771(8) \AA, c=19.0995(12) \AA, V=4462.8(5) \AA^{3}, Z=$ 
$4, \mu=0.575 \mathrm{~mm}^{-1}$. Of 63512 reflections collected, 12992 were independent; final $\mathrm{R}$ indices: $R_{1}=0.036$ (all data), $w R_{1}=0.090$ (all data).

Computational Details. All calculations were performed using the Gaussian98 software package on the Silicon Graphics Origin 2000 of the Vienna University of Technology. ${ }^{6}$ The geometry and energy of the model complexes and the transition states were optimized at the B3LYP level ${ }^{7}$ with the Stuttgart/Dresden ECP (SDD) basis set ${ }^{8}$ to describe the electrons of the ruthenium atom. For the $\mathrm{C}, \mathrm{N}$, and $\mathrm{H}$ atoms the $6-31 \mathrm{~g}^{\star *}$ basis set was employed. ${ }^{9} \mathrm{~A}$ vibrational analysis was performed to confirm that the structures of the model compounds have no imaginary frequency. Transition state optimizations were performed with the Synchronous Transit-Guided Quasi-Newton Method (STQN) developed by Schlegel et al. ${ }^{10}$ Frequency calculations were performed to confirm the nature of the stationary points, yielding one imaginary frequency for the transition states and none for the minima. The vibrational eigenvectors corresponding to the reaction coordinate (with imaginary frequency) of all transition states were visually checked to confirm the connectivity of transition states with the reactants and the products. Crucial transition states have been conformed by IRC calculations. All geometries were optimized without symmetry constraints. 


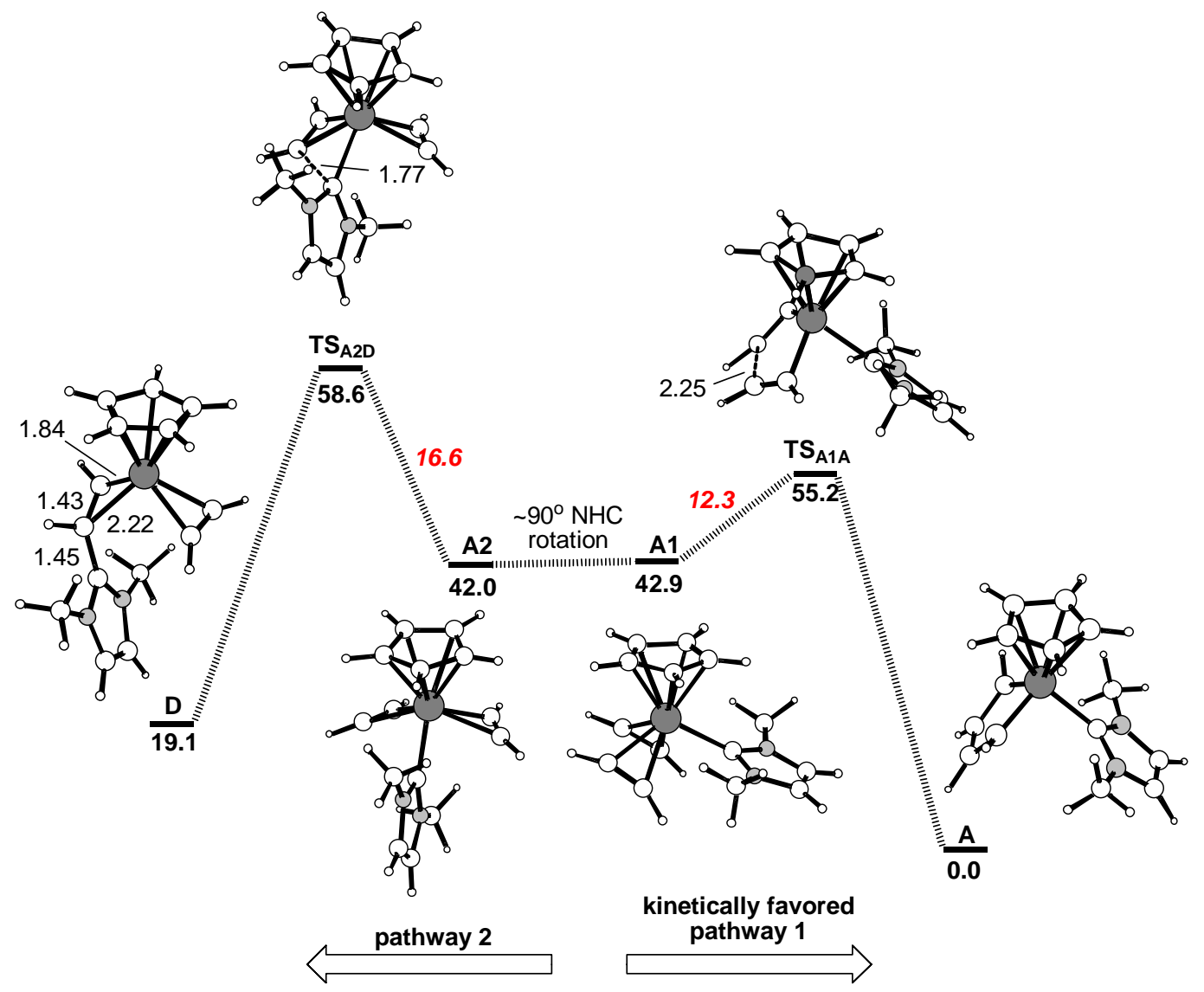

Figure S2. Reaction profile of the computed relative Gibbs free energies (in $\mathrm{kcal} / \mathrm{mol}$ ) for the migratory insertion of an acetylene ligand into the Ru-NHC bond ( $\mathrm{NHC}=1,3$-dimethylimidazol-2-ylidene) of the bisacetylene complex $\mathbf{A 2}$ to give the 1-metallacyclopropene intermediate (D) (bond distances in $\AA$ ).

\section{References}

(1) Perrin, D. D.; Armarego, W. L. F.Purification of Laboratory Chemicals, 3rd ed.; Pergamon: New York, 1988.

(2) (a) Kündig, E. P.; Monnier, F. R. Adv. Synth. Catal. 2004, 346, 901. (b) Gill; T. P.; Mann, K. R. Organometallics 1982, 1, 485.

(3) Jafarpour, L.; Stevens, E. D.; Nolan, S. P. J. Organomet. Chem. 2000, 606, 49.

(4) Bruker programs: SMART, version 5.054; SAINT, version 6.2.9; SADABS, version 2.10; XPREP, version 5.1; SHELXTL, version 5.1 (Bruker AXS Inc., Madison, WI, 2001).

(5) Sheldrick, G. M. SHELX97: Program System for Crystal Structure Determination; University of Göttingen, Göttingen, Germany, 1997. 
(6) Frisch, M. J.; Trucks, G. W.; Schlegel, H. B.; Scuseria, G. E.; Robb, M. A.; Cheeseman, J. R.; Zakrzewski, V. G.; Montgomery, J. A., Jr.; Stratmann, R. E.; Burant, J. C.; Dapprich, S.; Millam, J. M.; Daniels, A. D.; Kudin, K. N.; Strain, M. C.; Farkas, O.; Tomasi, J.; Barone, V.; Cossi, M.; Cammi, R.; Mennucci, B.; Pomelli, C.; Adamo, C.; Clifford, S.; Ochterski, J.; Petersson, G. A.; Ayala, P. Y.; Cui, Q.; Morokuma, K.; Malick, D. K.; Rabuck, A. D.; Raghavachari, K.; Foresman, J. B.; Cioslowski, J.; Ortiz, J. V.; Stefanov, B. B.; Liu, G.; Liashenko, A.; Piskorz, P.; Komaromi, I.; Gomperts, R.; Martin, R. L.; Fox, D. J.; Keith, T.; Al-Laham, M. A.; Peng, C. Y.; Nanayakkara, A.; Gonzalez, C.; Challacombe, M.; Gill, P. M. W.; Johnson, B. G.; Chen, W.; Wong, M. W.; Andres, J. L.; Head-Gordon, M.; Replogle, E. S.; Pople, J. A. Gaussian 98, revision A.7; Gaussian, Inc.: Pittsburgh, PA, 1998.

(7) (a) Becke, A. D. J. Chem. Phys. 1993, 98, 5648. (b) Miehlich, B.; Savin, A.; Stoll, H.; Preuss, H. Chem. Phys. Lett 1989, 157, 200. (c) Lee, C.; Yang, W.; Parr, G. Phys. Rev. B 1988, 37, 785.

(8) (a) Haeusermann, U.; Dolg, M.; Stoll, H.; Preuss, H. Mol. Phys. 1993, 78, 1211. (b) Kuechle, W.; Dolg, M.; Stoll, H.; Preuss, H. J. Chem. Phys. 1994, 100, 7535. (c) Leininger, T.; Nicklass, A.; Stoll, H.; Dolg, M.; Schwerdtfeger, P. J. Chem. Phys. 1996, 105, 1052.

(9) (a) McClean, A. D.; Chandler, G. S. J. Chem. Phys. 1980, 72, 5639. (b) R. Krishnan, J. S.; Binkley, R.; Seeger, J. A.; Pople, J. Chem. Phys. 1980, 72, 650. (c) Wachters, A. H. Chem. Phys. 1970, 52, 1033. (d) Hay, P. J. J. Chem. Phys. 1977, 66, 4377. (e) Raghavachari, K.; Trucks, G. W. J. Chem. Phys. 1989, 91, 1062. (f) Binning, R. C.; Curtiss, L. A. J. Comput. Chem. 1995, 103, 6104. (g) McGrath, M. P.; Radom, L. J. Chem. Phys. 1991, 94, 511.

(10) (a) Peng, C.; Ayala, P. Y.; Schlegel, H. B.; Frisch, M. J. J. Comp. Chem. 1996, 17, 49. (b) Peng, C.; Schlegel, H. B. Israel J. Chem. 1994, 33, 449. 